ORIGINAL ARTICLE

\title{
Feasibility and safety of day care laparoscopic cholecystectomy in a developing country
}

\author{
S Bal, L G S Reddy, R Parshad, R Guleria, L Kashyap
}

Postgrad Med J 2003;79:284-288

\begin{abstract}
Background: Although day care laparoscopic cholecystectomy (DCLC) has been shown to be safe in centres with adequate infrastructure for day care surgery, its feasibility and safety in developing countries has never been studied. Because of differences in the quality of health care delivery, western guidelines for day care surgery cannot be universally applied to developing countries.

Patients and methods: Patients less than 65 years who were graded I and II on the American Society of Anesthesiologists physical status score, irrespective of their educational status, living within 20 $\mathrm{km}$, and willing to make their own arrangements for a return to hospital in case of problems were selected for DCLC. Follow up was done by patients calling the hospital the morning after surgery. Results: $50 \%$ of the eligibility criteria were new; $313 / 383$ patients were suitable for DCLC. The commonest cause for rejection was that the patient lived out of the defined area (50\%). Altogether $92 \%$ were discharged within eight hours of surgery. The reasons for failure to discharge were the presence of abdominal drains in four $(2 \%)$, nausea and vomiting in nine $(3 \%)$, and conversion to open surgery in five $(2 \%)$. Ten patients (3\%) were readmitted; of these only two $(<1 \%)$ had complications needing re-exploration. Analysis of results showed that the inclusion and discharge criteria were valid and that the readmission and complication rates as well as the ease and accuracy of follow up were comparable to published data. DCLC reduced waiting times and increased patient turnover and may have a positive impact on resident training

Conclusions: DCLC is safe, feasible, and has potential benefits for health care delivery in developing countries. Each surgical service needs to develop their own guidelines based on local patient demography.
\end{abstract}

See end of article for authors' affiliations

Correspondence to: Associate Professor Sabyasachi Bal, AllMS,

Ansari Nagar, New Delhi

110029 , India;

drsbal@yahoo.com

Submitted 14 September

2002

Accepted

27 January 2003

$\mathrm{R}$ ecent publications have reported an increasing trend of traditional inpatient surgical procedures being performed as day care surgery, a development driven mainly by a desire to reduce health care expenditure without compromising the quality of care. ${ }^{12}$

Several published studies have testified to the safety and feasibility of day care laparoscopic cholecystectomy (DCLC). ${ }^{3-8}$ These reports, however, emanate from developed countries with well established norms for day care surgery with rigorously monitored outcomes. ${ }^{3-8}$ The reported safety of laparoscopic cholecystectomy cannot therefore be universally extrapolated to surgical practice everywhere.

Health authorities, quality assurance agencies, and surgeons in developing countries have traditionally been reluctant to advocate day care surgery because of major resource variations and inequity in quality of health care delivery. Consequently, there has been little or no attempt either to study the feasibility and safety of DCLC in these countries or to define viable recruitment criteria and determinants of acceptable outcomes of day care surgery.

Surgical groups in developing countries planning major day care surgery are thus severely constrained by the absence of published and validated models which can prospectively determine the safety of early discharge. Most centres have had to define their own methodology, using clinical trials. This is unsatisfactory because outcomes in clinical trials cannot always be predicted and may not be rigorously monitored.

This study describes the process of developing DCLC in a tertiary health care hospital of a developing country without a free standing facility or department for day care surgery and its successful integration into the regular surgical services of the hospital.

\section{PATIENTS AND METHODS}

Since we had no previous experience of DCLC as an outpatient procedure, a patient selection protocol suitable for our practice conditions was evolved. DCLC was offered to all patients with symptomatic, ultrasound proved gallstone disease who:

(1) Were less than 65 years, in sound mental health, and had a American Society of Anesthesiologists (ASA) physical status score ${ }^{9}$ grade I and II (patients with good control of their diabetes, chronic bronchitis, and hypertension were included).

(2) Lived within $20 \mathrm{~km}$ of the hospital.

(3) Had access to a telephone at all times and knew how to use it (it was not necessary that they had a rented line at home).

(4) Were living with a responsible adult and were capable of reaching the hospital on their own without depending on ambulance services, which are poorly developed in our region.

(5) Were thought to be able to understand instructions (this necessarily included the post-surgery primary care giver). No account was taken of the educational status in the selection of patients but the educational status was recorded.

(6) Agreed to the procedure as offered.

Patients with suspected bile duct stones on the basis of discriminant analysis of age, history, biochemistry, and radiology had preoperative endoscopic retrograde cholangiopancreatography. ${ }^{4}$ There was no other exclusion criteria.

No effort was made to avoid or exclude difficult gall bladders or patients with acute cholecystitis. Consultant

Abbreviations: ASA, American Society of Anesthesiologists; DCLC, day care laparoscopic cholecystectomy 


\begin{tabular}{|ll|}
\hline Table 1 Patient details \\
\hline Total patients operated (June 1997 to & 383 \\
June 2000) & 313 \\
Selected for outpatient surgery & 143 \\
ASA grade I & 148 \\
ASA grade II & 12 \\
ASA grade III (controlled & $14-65$ \\
hypertension/diabetes) & 33 \\
Age range & $70: 243$ \\
Median & \\
Sex (M:F) & \\
\hline
\end{tabular}

surgeons took informed consent and a departmental ethical clearance was obtained.

\section{Premedication and anaesthetic management}

A consultant anaesthetist saw all patients in the four weeks before surgery and assigned the ASA physical status score grade. Patients were given an information booklet outlining the procedure, potential problems, and details of perioperative care. Any change in status since the earlier evaluation was noted on the day of surgery using a predetermined proforma.

The patients were admitted at 08.00 hours on the day of surgery and were initially (first 50 patients) done as first patients on the list; later, the order in the list was variable provided the surgery was over by $3 \mathrm{pm}$. This allowed a clear five hours for observation before discharge. Although we have a designated recovery area, there are no nursing staff after $8 \mathrm{pm}$. All monitoring after this time has to be done by surgical residents; this was thought to be impractical for the purposes of this study.

No premedication was used. Intramuscular diclofenac 50 $\mathrm{mg}$ and metoclopramide (for the first 12 patients) or ondansetron $8 \mathrm{mg}$ was given intravenously before the start of anaesthesia as prophylaxis against postoperative pain, nausea, and vomiting. A standard anaesthetic protocol was used. ${ }^{4}$ A single dose ( $1.5 \mathrm{~g})$ cefuroxime prophylaxis was given intravenously at induction.

\section{Surgical procedure}

The study was undertaken in two phases. The first phase (99 patients) was a pilot project (for a postgraduate dissertation), and was completed in December 1998. In the second phase, outpatient laparoscopic cholecystectomy was offered as a routine service for all patients. The patient characteristics are shown in table 1 .

A standard four port procedure was used ${ }^{4} ; 0.5 \%$ bupivacaine was used to infiltrate port sites before incision. The initial 50 patients were operated by a consultant surgeon. Later, with increasing experience, surgery was routinely performed by trainees with consultant intervention only in difficult cases. Altogether 89 patients were operated by consultants and 190 by trainee surgeons; consultant intervention was necessary in a further 34 trainee operations because of difficulties or failure to make progress.

Routine drainage was not used; a closed suction drain was used when indicated. Insertion of a drain was not a contraindication for discharge provided other discharge criteria were met.

\section{Postoperative evaluation}

Immediately after surgery surgeons were required to record their assessment of the procedure, whether they anticipated any problem in the postoperative period, and whether the patients were fit to be discharged as was originally planned.

Patients were observed in a common recovery room. Parenteral diclofenac, metoclopramide, or ondansetron were used whenever indicated. Patients were assessed every 30 minutes by a member of the surgical team for pain, nausea, and vomiting. They were encouraged to sit up, drink as soon as possible, and to go to the toilet under supervision. An assessment chart, based on previously published literature, ${ }^{5}$ was used to assess the patient.

Patients were discharged if they were stable, fully conscious and if:

(1) The surgeon did not anticipate any problem from the operation.

(2) There was minimal nausea or vomiting.

(3) Pain was controlled or minimal.

(4) Patients were able to go the toilet without much difficulty (located about 25 metres from the recovery room).

(5) Patients were able to dress themselves.

(6) Patients were confident about going home.

Patients not satisfying any of the above criteria were admitted. Before discharge, all patients were given $50 \mathrm{mg}$ diclofenac intramuscularly and were prescribed diclofenac and metoclopramide or ondansetron tablets to be used on a regular basis for three days and then used only if required. Telephone numbers of the ward, the resident on call, and the consultant were provided. It was mandatory for the patient (or their care provider at home) to ring the consultant at home the next morning at a predetermined time to give a status report. A bed was kept vacant in the ward till patients phoned in (for the first 25 cases); the practice was later discontinued as experience and confidence improved. Waiting times for all patients undergoing outpatient cholecystectomy was calculated from the date of their first outpatient attendance. Simultaneously as an audit process, the waiting time for 126 patients undergoing DCLC between January 1996 to June 1997 was obtained.

Patients were reviewed at seven days. A published classification system was used to define complications. ${ }^{4}$ In the absence of any problems, patients were discharged from follow up. A patient satisfaction questionnaire was filled for all patients. The two tailed $t$ test for independent observations was used for analysis of data relating to waiting periods; $\mathrm{p}<0.05$ was taken as significant.

\section{RESULTS}

A total of 383 patients were evaluated for DCLC during the study period; 313 were found suitable (table 2). The mean waiting times for patients undergoing DCLC and inpatient procedure was 39 days (SD 9.6, range 9-63 days) and 136 days (SD 14.7, range 42-216 days) respectively ( $F$ test for equality of variance $=2.3447(125,312) \mathrm{df}, \mathrm{F}: 0.0000$; $\mathrm{T}$ for equal variances: $-81.3872 \mathrm{p} \leqslant 0.0000$; $\mathrm{T}$ for unequal variances: -68.4280 $\mathrm{p} \leqslant 0.0000)$.

One hundred and twelve patients (36\%) did not have personal telephones. In 126 patients $(40 \%)$, the primary care provider had schooling only until primary level.

Of the 313 patients, 290 patients (92\%) were discharged within 2-8 hours of the operation (median 4 hours). The

\begin{tabular}{|ll|}
\hline Table 2 & Reasons for unsuitability for \\
DCLC & \\
\hline Unsuitable ASA grade & 5 \\
Cancellation & $3^{*}$ \\
Age greater than 65 & 6 \\
Unsuitable social conditions & 10 \\
Lived out of defined area & 35 \\
Suspected CBD stones & 7 \\
Patient preference & 4 \\
\hline *Three patients initially cleared during first \\
evaluation were found to have uncontrolled \\
hypertension; one also had arrhythmias. \\
CBD, common bile duct. \\
\hline
\end{tabular}


Table 3 Failure to discharge-causes

\begin{tabular}{lll}
\hline Reasons for failure to discharge & $\begin{array}{l}\text { First phase } \\
(n=100)\end{array}$ & $\begin{array}{l}\text { Second phase } \\
(n=213)\end{array}$ \\
\hline Conversion to open surgery & 2 & 3 \\
Insertion of drains & 2 & 2 \\
Unexplained pain and tachycardia & 0 & 1 \\
Nausea and vomiting & 5 & 4 \\
Patient refusal & 2 & 0 \\
\hline
\end{tabular}

Table 4 Reasons for readmission

\begin{tabular}{lll}
\hline Reasons for readmission & No & Outcome \\
\hline Pain and abdominal distension & 8 & $6(75 \%)$ discharged in 24 hours \\
Features of peritonitis & 2 & Both re-explored: case 1, biliary \\
& $\begin{array}{l}\text { peritonitis (discharged after 56 } \\
\text { days); case 2, iatrogenic } \\
\text { duodenal perforation } \\
\text { (discharged after } 8 \text { days) }\end{array}$ \\
\hline
\end{tabular}

reasons for failure to discharge are shown in table 3. Six of the $10(60 \%)$ patients with drains were discharged with drains in situ. These were removed after 48 hours in the ward. The remaining four with drains were admitted for observation (table 3). Three were discharged the next morning; one patient, complaining of unexplained pain and tachycardia, was discharged after three days. Five patients $(2 \%)$ were converted and an open cholecystectomy was performed. They were discharged between three to five days after surgery. Nine patients $(3 \%)$ could not be discharged due to severe nausea or vomiting; they were discharged next morning.

Eight patients (3\%) were readmitted three to five days later with pain and abdominal distension. Six of these eight (75\%) recovered on conservative management and were discharged 18-72 hours after readmission. Two patients needed reexploration ( $1 \%)$. One patient was explored for biliary peritonitis secondary to a leak from the cystic duct stump due to a slipped clip. A common bile duct stone was also found on operative cholangiography. The stone was removed and the cystic duct controlled. The patient needed ventilation and intensive care and was discharged 56 days later. The second patient had a duodenal perforation secondary to diathermy injury. This was repaired with an omental patch; the patient made an uneventful recovery and was discharged eight days later (table 4). A review indicated that both had met our DCLC discharge criteria. Seventeen $(6 \%)$ patients telephoned the hospital for what they thought was inappropriate pain. In all cases, the symptoms were assessed as not serious by the surgeon who took the call. All were well at follow up.

\section{DISCUSSION}

Day care surgery is possibly the clearest and most evident example of economy in any health care system. ${ }^{12}$

Evidence supporting safety of major DCS including laparoscopic cholecystectomy is predominantly composed of case series of selected patients..$^{3-8}$ Universal extrapolation of published experience is potentially dangerous because poor and unacceptable outcomes have been reported. ${ }^{10}{ }^{11}$ The risk may be even more in economically deprived countries where low literacy rates, lack of reliable and efficient transport, absence of organised referral patterns, poorly developed communication systems, underdeveloped primary health care services, and absence of community nursing have prevented the successful introduction of major surgical operations as day care surgery. Unfortunately, these are the very countries that could benefit most from day care surgery by reducing health care expenditure and waiting times.

A major problem has been the absence of safe guidelines. There was little published experience of day care surgery from developing countries until recently. Existing experience relates to practice in private health care centres where facilities are in accordance with published western guidelines. $^{1213}$ A centre without previous experience, and without infrastructure to support major day care surgery, needs to evolve well reasoned guidelines with a rigid and honest appraisal of outcomes to identify deficiencies and potential pitfalls. The absence of malpractice litigation in our surgical practice puts an even greater responsibility on those advocating major day care surgery. Table 5 summarises our results and compares them with the experience reported in the literature. ${ }^{3-8}$

The viability of our inclusion criteria and the feasibility of day care surgery in our practice was analysed using successful discharge, readmission and complication rates, ease and accuracy of follow up, impact on resident training, and the potential benefit to our surgical service from day care surgery based on DCLC as a model was examined.

Eighty two percent of patients met the criteria for eligibility. Of those found unsuitable, 50\% (36/70) were rejected only because they lived out of the defined area. This is common in developing countries and highlights a major problem in the organisation of day care surgery where there are no defined referral territories and where patients often have to travel to centres with better infrastructure, resources, and "reputation".

Ninety two percent of patients were successfully discharged thus superficially validating our recruitment criteria. Three of our entry criteria (criteria 2, 3, and 4) are new and have not been reported previously.

The choice of a $20 \mathrm{~km}$ radius as the outer limit for entry to our study was arbitrary. This is obviously a variable that depends on economic resources, existing transport services, and the geography of the terrain and our criterion is merely illustrative and intended to serve only as a guideline and/or methodological tool. Though the onus of reaching the hospital

\begin{tabular}{|c|c|c|c|c|c|c|}
\hline \multicolumn{7}{|c|}{ Table 5 Comparative results } \\
\hline Series & $\begin{array}{l}\text { Total } \\
\text { operated }\end{array}$ & $\begin{array}{l}\text { No } \\
\text { undergoing } \\
\text { DCLC }\end{array}$ & $\begin{array}{l}\text { Patients } \\
\text { eligible for } \\
\text { DCLC (\%) }\end{array}$ & $\begin{array}{l}\text { Successful } \\
\text { discharge } \\
(\%)\end{array}$ & $\begin{array}{l}\text { Readmission } \\
(\%)\end{array}$ & $\begin{array}{l}\text { Complications } \\
\text { (\%) }\end{array}$ \\
\hline Smith et al ${ }^{6}$ & 266 & 98 & 36 & 81 & - & - \\
\hline Prasad and Foley ${ }^{5}$ & 103 & 51 & 49 & 92 & - & $12^{*}$ \\
\hline Lillemoe $^{3}$ & NS & 130 & NS & 94 & 4.6 & - \\
\hline Fiorillo et aP & 238 & 149 & 63 & 61 & - & 2.1 \\
\hline Stephenson et a ${ }^{\beta}$ & NS & 15 & NS & 80 & - & - \\
\hline Mialand et $a^{4}$ & NS & 200 & NS & 94 & 8 & 7 \\
\hline Present series & 383 & 313 & 82 & 93 & 3.4 & $<1 t$ \\
\hline
\end{tabular}


for perioperative problems was on the patient, our results suggest a satisfactory outcome if patients and relatives understand the issues related to day care surgery and the response expected from them. It also appears that domiciliary perioperative care, even in our set-up, is feasible and reliable. Provided the pathways of care are clearly defined and understood, similar results can probably be achieved in most situations. In this context, our selection criteria appear to be both valid and practical.

Our study can be criticised on the grounds that patients were exposed to unwarranted risks by use of criteria that had not been validated. This criticism is valid and we concede that there should be better ways of undertaking feasibility studies. Unfortunately when we began the study, there were no objective methods or models available to determine the safety of early discharge. A recent publication has illustrated the use of a simulation model to study transition of care. ${ }^{2}$ This model has now been validated ${ }^{3}$ and in future, it might be more appropriate to use the model before adopting day care surgery as routine, especially where experience is small. It must be pointed out, however, that even minimally literate patients respond satisfactorily to the demands and responsibilities of day care surgery and should not be eliminated for this reason alone.

Cancellation on the day of surgery is an indirect indicator of the quality of day care services. Besides causing inconvenience to patients, it adversely affects surgical schedules (thereby annulling the benefits of day care surgery). In addition, a high cancellation rate suggests deficient initial preanaesthetic evaluation, which is potentially dangerous. Fewer than $1 \%$ of patients had their operation cancelled on the day of surgery. A few cancellations are acceptable because new clinical situations may arise after the earlier evaluation, especially if there is a significant time between evaluation and surgery; this happened in three of our patients. Since such changes in clinical status jeopardise patient safety, a close watch needs to be kept for comorbid conditions that are known to change (for example, hypertension) over short periods. Further, alteration of social conditions in the waiting period must also be looked into. These issues have not been adequately addressed in published literature; also, the optimal interval between preoperative anaesthetic evaluation and surgery is uncertain. It has been recently suggested ${ }^{14}$ that this could be up to 30 days for low risk patients and this is supported by our own experience. For high risk patients being considered for day care surgery, the evaluation probably needs to be done as close to surgery as feasible. There are no studies which have examined this issue and definite recommendations do not exist. While successful day care surgery has been reported in high risk patients, ${ }^{36}$ the degree of selection exercised in these studies is uncertain. In most cases, and certainly in conditions like ours, it is probably better to limit day care surgery to ASA grade I and II patients.

Unplanned admission after day care surgery is an indicator of quality assurance ${ }^{15}{ }^{16} ; 3 \%$ of our patients needed readmission. However, only $2 / 290$ patients $(<1 \%)$ required further surgery. Both these patients developed complications five days after discharge and were unlikely to have been examples of missed diagnosis during initial discharge. Similar experience has been reported in the literature ${ }^{4}$ and is not a specific problem of day care surgery. Our criteria for discharge are therefore, satisfactory; the discharge parameters, based on published criteria, also appear to be reliable. ${ }^{5}$

Follow up by telephone is the commonest and most cost effective method after day care surgery. If published guidelines were to be rigidly followed, countries with poor telecommunication infrastructure would necessarily have to restrict the ambit of day care surgery, negating its impact on health care delivery. Our data clearly show that follow up can be equally reliable if patients are asked to telephone the hospital rather than the traditional method of medical teams contacting patients.

During the initial part of the study (first 45 patients) consultants assessed patients after day care surgery using a structured proforma, but as experience grew residents took over this job. All patients ( $\mathrm{n}=17 ; 6 \%$ ) who called the hospital with perceived problems were satisfactorily assessed using a structured interview. Structured forms have been shown to be a better assessment tool compared with narrative notes and they also help in training medical personnel. ${ }^{17}$

The most important benefit of day care surgery is reduction in health care expenditure but benefits are not necessarily similar for all countries. The cost of surgery in developing countries is low vis a vis those in developed countries. An expectation of reduction in costs would be rational but our data do not permit us to project a categorical benefit. There may, however, be another definite advantage of day care surgery that is relevant to surgical practice in developing countries as described below.

The number of surgical beds in any hospital, including ours, are relatively constant and non-flexible. Since our region has a high prevalence of gallstones, a large number of beds are used for cholecystectomy. This causes delays in the care of patients with more serious medical problems such as malignancies and emergencies. Additionally, many (up to $60 \%$ ) patients are from outside the proposed geographical area of our hospital. Repeated journeys to the hospital for evaluation and therapy increases cost of health care tremendously. Inpatient evaluation is often the most efficient approach to diagnosis and therapy in these groups but can often run into problems since beds may be blocked by patients with benign but widely prevalent surgical disease. Some prioritisation occurs covertly (as shown by the long waiting period for patients undergoing inpatient cholecystectomy) to favour more serious illnesses but this may be unfair to patients with benign diseases like gallstones, because it delays treatment. Benign diseases often occur in those who are relatively young, who are in the most productive period of life, and who may have young dependents. Moreover, symptoms and complications of benign diseases including gallstones can be severe or even life threatening. It is therefore necessary to achieve an optimal balance without compromising care of groups with more complex disease. Our study has shown reduction in waiting times for patients undergoing day care surgery compared with inpatient cholecystectomy. The study has also transferred a large number of patients with gallstones (who would otherwise be treated as inpatients) to day care surgery thereby leading to better bed utilisation. This has also been the experience of other groups. ${ }^{1}$

This outcome is theoretically beneficial but it is not a long term solution to the complex issue of equitable distribution of acceptable health care for the two groups and problems are bound to arise in the future. It is the recognition of this future problem that makes the results of our study so important. Our results should encourage additional studies to expand the list of procedures that can be brought under the umbrella of day care surgery. It should also provide health authorities with viable options while planning solutions and act as evidence based impetus for the development of exclusive day care facilities. In this way, the management of both simple and complex health problems could receive equitable attention and would become more efficient.

The last issue concerns surgical training. Objections have been raised about several aspects of training where day care surgery has been used. ${ }^{18}{ }^{19}$ It is our belief that maintaining the dual objective of ensuring patient safety while training residents is feasible and training can proceed unaffected in a well supervised programme of day care surgery. Sixty one percent of the operations in the study were performed by residents with similar outcomes to those performed by consultants. Residents also participated in evaluation, perioperative assessment, and follow up of patients. This demonstrates that training and acquisition of operative experience need not suffer in active day care programmes and that training and ensuring patient safety are not mutually exclusive. 
There is now carefully reviewed evidence that resident training is satisfactory in a well defined training programme where laparoscopic cholecystectomy and day care surgery have been well integrated into training objectives. ${ }^{20}{ }^{21}$ Planning of services and resource management are going to be the dominant issues of the future and an efficient day care surgery programme will provide invaluable training in the planning of guidelines, assessment of patients, and use of alternative methods of follow up. This will lead to better surgical decision making and acquisition of management skills in scenarios different from traditional inpatient environment.

\section{Authors' affiliations}

S Bal, L G S Reddy, R Parshad, R Guleria, L Kashyap, All India Institute of Medical Sciences, New Delhi, India

\section{REFERENCES}

1 Boothe P, Finegan BA. Changing the admission process for elective surgery: an economic analysis. Can J Anaesth 1998;42:391-4.

2 Fleisher LA, Yee K, Lillemoe KD, et al. Is outpatient laparoscopic cholecystectomy safe and cost effective? A model to study transition of care. Anesthesiology 1999;90:1746-55.

3 Lillemoe KD. Laparoscopic cholecystectomy as a "true" outpatient procedure: initial experience in 130 consecutive patients. J Gastrointest Surg 1999;3:44-9.

4 Mialand O, Raeder J, Aasboe V, et al. Outpatient laparoscopic cholecystectomy. BrJ Surg 1997:84:958-61.

5 Prasad A, Foley RJE. Day case laparoscopic cholecystectomy: a safe and cost effective procedure. Eur J Surg 1996;162:43-6.

6 Smith R, Kolyn D, Pace R. Outpatient laparoscopic cholecystectomy. HPB Surg 1994;7:261-4
7 Fiorillo MA, Davidson PG, Fiorillo JA, et al. 149 day care laparoscopic cholecystectomies. Surg Endosc 1996;10:52-6.

8 Stephenson BM, Callander C, Sage $M$, et al. Feasibility of "day case" laparoscopic cholecystectomy. Ann R Coll Surg Engl 1993;75:249-51.

9 American Society of Anaesthesiologists. New classification of physical status. Anesthesiology 1963;24:11-7.

10 Schloss MD, Tan AK, Schloss B, et al. Outpatient tonsillectomy and adenoidectomy: complications and recommendations. Int J Pediatr Otorhinolaryngol 1994:30:1 15-22.

11 Mitchell RB, Pereira KD, Friedman NR. Outpatient tonsillectomy. Is it safe in children younger than age 13? Arch Otolaryngol Head Neck Surg 1997;123:681-3

12 Ramanujam TM, Uma G, Usha V, et al. Advantages and limitations of day surgery in children in a developing country. Pediatr Surg Int 1998;13:512-4.

13 Mandhan P, Shah A, Khan AW, et al. Outpatient pediatric surgery in a developing country. Journal of the Pakistan Medical Association 2000;50:220-4.

14 Pollard JB, Olson L. Early outpatient preoperative anaesthesia assessment: does it help to reduce operating room cancellations? Anesth Analg 1999:89:502-5.

15 Margovsky A. Unplanned admissions in day-case surgery as a clinical indicator for quality assurance. Aust N Z J Surg 2000;70:2 16-20.

16 Twersky R, Fishman D, Homel P. What happens after discharge? Return hospital visits after DCS. Anesth Analg 1997;84:319-24.

17 Kleinpell RM. Improving telephone follow-up after DCS. Journal of Perianaesthesia Nursing 1997:12:336-40.

18 Shaper NJ, Harrison M, Bates T. Impact of laparoscopic cholecystectomy on surgical training. Ann R Coll Surg Engl 1996;78:39-42.

19 Namias N, McKenney MG, Sleeman D, et al. Trends in resident experience in open and laparoscopic cholecystectomy. Surg Laparosc Endosc 1997; 7:245-7.

20 Schol FP, Go PM, Gouma DJ, et al. Laparoscopic cholecystectomy in a surgical training programme. Eur J Surg 1996;162:193-7.

21 Bockler D, Geoghegan J, Klein M, et al. Implications of laparoscopic cholecystectomy for surgical residency training. JSLS 1999;3:19-22. 